\title{
Data Origin
}

National Cancer Institute

\section{Source}

National Cancer Institute. Data Origin. NCI Thesaurus. Code C103167.

The primary point where the data was generated or collected. 